Revue d'histoire de l'Amérique française

ATS REVUE D.HISTOIRE DE L'AMÉRIQUE FRANÇAISE

\title{
La rencontre de la théologie et des sciences sociales au Québec
}

\section{Gilles Routhier}

Volume 57, numéro 3, hiver 2004

URI : https://id.erudit.org/iderudit/009595ar

DOI : https://doi.org/10.7202/009595ar

Aller au sommaire du numéro

Éditeur(s)

Institut d'histoire de l'Amérique française

ISSN

0035-2357 (imprimé)

1492-1383 (numérique)

Découvrir la revue

Citer cet article

Routhier, G. (2004). La rencontre de la théologie et des sciences sociales au Québec. Revue d'histoire de l'Amérique française, 57(3), 389-405.

https://doi.org/10.7202/009595ar d'utilisation que vous pouvez consulter en ligne.

https://apropos.erudit.org/fr/usagers/politique-dutilisation/ 


\section{La rencontre de la théologie et des sciences sociales au Québec}

GILLES ROUTHIER

Faculté de théologie

Université Laval

$\mathbf{L}$

ES RELATIONS entre théologie et sciences sociales ont connu diverses périodes et on peut observer une certaine évolution pendulaire entre des positions tantôt fusionnelles et tantôt polémiques ${ }^{1}$. Ainsi, à la fin du $\mathrm{XIX}^{\mathrm{e}}$ siècle, la sociologie de la religion, au moins en France, «s'était coulée dans le mouvement laïque», comme le rappelait François-A. Isambert ${ }^{2}$. Aussi, en 1954, lors de la constitution du Groupe de sociologie des religions et du lancement, la même année, des Archives de sociologie des religions, Joachim Wach plaidait "pour qu'à l'affectation d'un coefficient antireligieux ne succède pas une annexion de la sociologie religieuse par les confessions chrétiennes ${ }^{3}$ ».

La situation française n'est toutefois pas représentative du climat général qu'on pourrait résumer, de manière trop schématique certes, dans les lignes suivantes : après la période euphorique qui suivit le concile Vatican II qui avait fait appel à des sociologues et qui avait encouragé l'usage de la

1. Même s'il y a d'indéniables différences, on peut observer des parallèles entre la rencontre entre la théologie et les sciences sociales et celle de la philosophie et des sciences sociales au Québec. Voir Louise Marcil-Lacoste, «Le regard de l'autre : la philosophie et l'émergence des sciences sociales", dans Continuité et rupture. Les sciences sociales au Québec (Montréal, Presses de l’Université du Québec, 1984), 435-454.

2. F.-A. Isambert, "Quarante ans déjà... », Archives de sciences sociales des religions, 93 (1996) : 20.

3. Ibid.,18. 
sociologie dans la formation des séminaristes ${ }^{4}$, période davantage marquée par un engouement pour la sociologie pastorale que pour la sociologie de la religion, les rapports entre théologie et sociologie se sont à nouveau brouillés à la fin $\mathrm{du} \mathrm{xx}^{\mathrm{e}}$ siècle, comme en témoigne le débat récent autour de l'ouvrage de John Milbank 5 .

Pour le Québec ${ }^{6}$, c'est ce qui m’intéressera désormais, on peut probablement décrire un itinéraire en quatre moments.

\section{AU FONDEMENT DU CATHOLICISME SOCIAL : DES THÉOLOGIENS APPRENTIS SOCIOLOGUES}

La sociologie rencontre d'abord la théologie par le biais de l'analyse de la société, et ce, dès la fin de la deuxième moitié du xix ${ }^{\mathrm{e}}$ siècle au moment où l'industrialisation prend racine en Europe et que se profile l'affrontement entre le capitalisme et le socialisme. Ici, l'influence du catholicisme social européen, et celle de Frédéric Le Play en particulier ${ }^{7}$, est déterminante. Du point de vue méthodologique, celui-ci est considéré comme un des fondateurs de la sociologie expérimentale ou, mieux, de la sociographie. Son livre phare, Les ouvriers européens (1855), illustre bien sa méthode. Devant le succès de son ouvrage couronné par l'Académie des sciences (1856), Le Play fonde la Société d'économie sociale qui poursuivra des recherches sur la condition des ouvriers et en assurera la publication sous le titre Ouvriers des deux mondes dont deux séries d'études de cinq volumes chacune sont publiées entre 1857 et 1899 . On y retrouve des monographies de familles ouvrières d'Asie, d'Afrique et d'Amérique. C'est dans la troisième série de ces études, en 1904, que Stanislas A. Lortie publiera son étude Compositeur typographe de Québec qui présente une

4. Voir le décret Optatam totiuss, paragraphe 2 C. Par ailleurs, la rédaction de la constitution Gaudium et spes sur l'Église dans le monde de ce temps avait fait appel à l'expertise de nombreux sociologues.

5. John Milbank, Theology and Social Theory. Beyond Secular Reason (Oxford, Blackwell, 1991), et Robin Gill, dir., Theology and Sociology. A Reader (London, Cassell, 1996). Voir en particulier la quatrième partie où les thèses de Milbank sont mises en discussion. Celui-ci déniait aux sciences sociales leur rôle dans la construction de la réflexion théologique et dénonçait l'importance qu'elles y avaient prise.

6. L'article fera appel à diverses expériences, plusieurs exemples cependant se rapportant à la ville de Québec, mais sans s'y limiter.

7. Sur l’influence de Le Play au Canada, voir Pierre Trépanier, «Les influences le playsiennes au Canada français, 1855-1888», Revue d'études canadiennes, XXII,1 (1987) : 66-83; du même, «Le Québec à l'école de Le Play", Sociétés, 23 (1989) : 18-19. On verra également Jean-Philippe Warren, L'engagement sociologique. La tradition sociologique du Québec francophone (18886-1955) (Montréal, Boréal, 2003). 
famille ouvrière de la paroisse Saint-Jean-Baptiste de Québec. C’est qu'avec plusieurs autres, Alphonse Desjardins, Paul de Cazes, Louis-Amable Jetté, etc., Stanislas Lortie était devenu membre, depuis les années 1890, de la Société d'économie sociale fondée par Le Play. C'est d'ailleurs sur son modèle que Lortie fondera, en 1905, à l'Université Laval, la Société d'économie sociale et politique de Québec ${ }^{8}$ à laquelle est naturellement associé Paul-Eugène Roy, figure emblématique du catholicisme social de l'époque'.

Avec Le Play et ses descendants, on est en présence d'une méthode qui fera école, méthode empirique qui s'intéresse à l'observation des réalités sociales et qui représente le premier point de contact sans doute entre la théologie et les sciences sociales. Ici, toute la réflexion se construit à partir d'observations colligées systématiquement, ce qui déplace de manière significative la façon dont se construisait la doctrine catholique de cette époque. Il ne faut pas négliger cependant que l'on est aussi en présence d'une théorie ou d'une vision de la société, certains ont même parlé à ce sujet d'une doctrine de la société.

C'est sans doute à ce chapitre que divergent le monde des théologiens et celui des sociologues. Le Play, comme ses successeurs et comme le fera largement la doctrine sociale catholique, part d'une vision du monde qui comporte, comme clé de voûte, le concept d'ordre ${ }^{10}$ ou d'harmonie, réalités qu'il croit trouver dans la nature ou dans la création. Cette harmonie a été détruite par l'introduction de désordres (à son époque, les révolutions, le développement du capitalisme et l'apparition du communisme). De là, Le Play postule que la paix sociale repose sur un retour à l'ordre qui passe par le rétablissement de la morale et la restauration du principe d'autorité aussi bien au sein de la famille, qu'à l'usine, dans le pays et dans l’Église. Comme ses successeurs - ce sera le cas chez Mounier - la crise n'est pas simplement ou d'abord d'ordre économique, elle est avant tout morale puisqu'elle provient d'un désordre ou d'une conduite déréglée. Le

8. Il existait à Montréal une société du même type. Voir P. Trépanier, «La Société canadienne d'économie sociale de Montréal, 1888-1911 : sa fondation, ses buts et ses activités ", Canadian Historical Review, LXVII,3 (1986) : 343-367.

9. Sur S. Lortie, voir Pierre Savard, "Lortie, Stanislas», Dictionnaire biographique du Canada (Québec, Les Presses de l'Université Laval, 1998), XIV : 720. Plus récemment, Jacques Racine, "Citoyen de Québec, citoyen du monde : Stanislas Lortie», dans Gilles Routhier et Jean-Philippe Warren, dir., Les visages de la foi. Figures marquantes du catholicisme québécois (Montréal, Fides, 2003), 147-159.

10. Voir Gilles Routhier, «L'ordre du monde. Capitalisme et communisme dans la doctrine de l’École sociale populaire, 1930-1936», Recherches sociographiques, XXII,1 (1981) : 7-48. 
sous-titre de son ouvrage de 1855 est d'ailleurs très symptomatique à cet égard : "Études sur les travaux, la vie domestique et la condition morale des populations ouvrières de l'Europe, précédées d'un exposé de la méthode d'observation".

On le voit, le sociologique et le théologique opèrent ici un certain nombre de transactions puisque les théories qui commandent la compréhension du monde sont ici clairement théologiques ou morales. Elles s'appuient sur une vision du monde qui renvoie à une "doctrine» de la création. Bien plus, les réalités sociales en présence ne sont pas closes sur elles-mêmes, mais ouvertes sur une référence spirituelle et théologique. Réformistes, ces «sociologues chrétiens» font appel aussi bien au «jugement de fait» qu'au «jugement moral».

L'École sociale populaire ${ }^{11}$, dont la fondation en 1911 s'inspire de l'Action populaire de Reims (1903) et qui fera vite concurrence à sa devancière de Québec, l'Action sociale catholique, représente aussi un milieu où se multiplient les transactions entre une pratique d'enquête sociale et la doctrine catholique. Il s'agit sans doute de l'illustration la plus achevée de ce type de rapport entre théologie et les sciences sociales. De 1910 à 1950, on trouve là le foyer le plus important de rencontre entre la doctrine catholique et la pratique des sciences sociales. Conçue comme un carrefour, l'ESP est à la fois un centre de formation et d'étude de la doctrine sociale de l'Église, un lieu de recherche et d'enquête sociale et une base de propagande (conférences, journaux, brochures, tracts, etc.).

Les années de crise (1930-1936), dominées par l’activité du père Joseph Papin-Archambault, en marqueront l'apogée. Les évêques, craignant que les ébranlements de la société provoqués par le chômage ouvrent la voie au communisme et au socialisme, veulent proposer un véritable projet de société pouvant concurrencer celui du CCF dont il ne suffit pas de réfuter le programme pour fournir une solution de rechange au programme des vieux partis compromis avec les trusts. Leur Lettre pastorale à l'occasion du malaise économique des temps présents (1932) et celles de 1933 indiquent leur volonté de dépasser la seule dénonciation du socialisme et du communisme. Devant les demandes répétées des catholiques, il devient évident que seul un contre-programme est en mesure d'ouvrir une nouvelle voie. J. Papin-Archambault convoque alors, le 9 mars 1933, treize ecclésiastiques au scolasticat de l’Immaculée-Conception. La discussion

11. En plus de mon article en note précédente, voir mon mémoire de maitrise La doctrine sociale et le mouvement catholique : l'École sociale populaire (1930-1936), Université Laval, 1979. 
conduit à la rédaction d'un programme de restauration sociale en treize articles. Il s'agit avant tout d'un manifeste à caractère doctrinal qui, sans entrer dans les détails de l'organisation corporative, ébauche une législation sociale, propose la réglementation des opérations financières et envisage quelques mesures de contrôle de la gestion publique. Des mesures concrètes sont avancées : allocations au chômage, pensions de vieillesse, salaire familial pour les ouvriers, législation sur les risques d'accident et de maladie, etc. Par-dessus tout, il appuie le corporatisme non étatique promu par Pie XI et fondé sur la coopération entre associations de patrons et ouvriers œuvrant dans une même branche de la production.

Peu de temps après, un groupe de laïcs, réunis sous les auspices de l'ESP, publie un second programme, plus détaillé et plus technique. On y aborde la réforme morale, la restauration rurale, la question ouvrière, la lutte contre les trusts et finalement les réformes financières et politiques. Bien accueilli par Le Devoir, L'Action nationale et L'Action catholique, ce programme est par la suite emprunté par l'Action libérale nationale, puis l'Union nationale, voire par les libéraux, lors de l'élection de 1936.

C'est là le sommet atteint par ce mouvement de restauration sociale. Dès que l'on sort de la Crise et que l'on approche de la Deuxième Guerre mondiale, cette logique montre des signes d'épuisement. La Lettre pastorale sur la restauration de l'ordre social (11 mars 1941) constitue le baroud d'honneur d'une période qui s'achève.

Au fur et à mesure qu'on avance vers le milieu du siècle, on trouve d'autres initiatives qui prolongent autant qu'elles dépassent les premières manifestations du catholicisme social marquées par le corporatisme et l'idéologie clérico-nationaliste et de restauration dans lesquels s'étaient moulées l'Action sociale catholique à Québec et l'École sociale populaire, à Montréal. L'un des foyers qui a fait le plus de bruit au cours de la décennie 1950 est sans doute la Commission sacerdotale d'étude sociale (19471960) qui est de toutes les grandes entreprises de révision de la pensée sociale au Québec au cours de cette période, spécialement à la suite de la grève de l'amiante. Elle est manifestement apparentée à l'un des regroupements les plus fameux de cette époque, le Centre de culture populaire de l'Université Laval, animé par l'abbé Gérard Dion. Licencié en théologie (1939), détenteur d'une maîtrise en sciences sociales (1943) et professeur de relations industrielles, l'abbé Dion publie, à partir de 1947, Ad usum sacerdotum, bulletin mensuel destiné aux prêtres œuvrant dans le domaine de l'action sociale et qui sera bientôt connu sous le titre Perspectives sociales. Le Centre de culture populaire, au sujet duquel on ne possède pas encore 
de monographie, regroupe des catholiques qui ont une pratique des sciences sociales au sens large (Gérard Dion, Fernand Dumont, GeorgesHenri Lévesque, Louis O’Neill, etc.). À travers le bulletin qu'ils publient, c'est une autre manière de voir le Québec qui s'élabore et une autre façon de penser le rôle de l'Église dans la société qui se construit. Il ne faut pas non plus négliger l'apport considérable de l'École des sciences sociales de l'Université Laval avec le père Georges-Henri Lévesque ${ }^{12}$ qui forme, avec l'unité d'enseignement en relations industrielles et une autre en service social (où l'on compte un plus grand nombre de femmes), un ensemble qui s'intéresse aux pratiques sociales. Les idées reçues sont graduellement en cours de révision. De nouvelles méthodes et de nouveaux instruments pour analyser la réalité conduisent à l'élaboration d'un autre discours, à des prises de conscience rajeunies et à la proposition de solutions novatrices. Ces praticiens des sciences sociales sont des passeurs de frontières ou des théologiens des marges qui assurent une interface entre théologie et sciences sociales, sans que l'on sache toujours quand s'arrête l'œuvre du théologien et quand commence celle du second. C'est par les pratiques sociales en émergence et une affinité avec la mentalité scientifique que ces ecclésiastiques se mettent à fréquenter les sciences sociales. On a ici affaire, suivant l'expression de Henri Desroches, à une "socio-théologie» ou à une «théologie sociale, sociologisante ou sociologisée $e^{13}$ ».

À côté de ces lieux de réflexion, on remarque la présence de l'école jésuite (avec, en particulier, les pères Arès et Cousineau, qui ont pris le relais du père Papin-Archambault), autour de la revue Relations, et celle de l'école dominicaine, autour de la revue Maintenant qui prend son envol avec la Révolution tranquille.

Proche, en un certain sens du catholicisme social, mais s'en distinguant tout de même, l'Action catholique représente un autre lieu de contacts entre la théologie et la sociologie. Comme le notera André-J. Bélanger, au Québec, la JEC a été «la première expérience de distanciation d'avec le clergé, à l'intérieur même de l'encadrement ecclésial ${ }^{14}$ » et cette distanciation n'est pas étrangère à l'adoption d'une autre manière de construire la réflexion. Non seulement les militants d'Action catholique prennent-ils

12. Pour un aperçu de son rayonnement, on lira Robert Parisé, Georges-Henri Lévesque, père de la renaissance québécoise (Montréal, Alain Stanké, 1976).

13. Henri Desroches, "Des sociologies religieuses à une sociologie des religions", dans Sociologies religieuses (Paris, Presses universitaires de France, 1968), 20.

14. A.-J. Bélanger, Ruptures et constantes. Quatre idéologies du Québec en éclatement : la Relève, la JEC, Cité libre, Parti Pris (Montréal, Hurtubise HMH, 1977), 36. 
l'habitude de lire en groupe l'Écriture sainte, mais on trouve dans ces milieux tout un apprentissage d'une réflexion autonome, qui ne s'appuie pas sur les réponses toutes faites ou les énoncés préfabriqués. Les discussions en équipe permettent un apprentissage du débat public puisque l'on est amené à exprimer ses vues et à les confronter à celles des autres. Pardessus tout, les militants apprennent à réfléchir de manière inductive. $\mathrm{La}$ première démarche de tout militant d'Action catholique est de «voir». Ce qu'on leur demande avant tout c'est d'observer la réalité, de noter des faits, des faits bien circonscrits, minutieusement décrits. Leur premier instrument, c'est leur carnet de faits. Enfin, toutes discussions en équipe d'Action catholique procèdent à partir d'une analyse et d'une interprétation de la réalité. Cela est au fondement de la méthode «Voir - Juger - Agir».

\section{LA SOCIOLOGIE PASTORALE : L'HEURE DES SOCIOLOGUES}

En 1951, dans le cadre de la troisième Conférence internationale de sociologie religieuse, Jean-Charles Falardeau signalait "qu'on ne trouve, au Canada français, aucune étude approfondie des pratiques religieuses des populations rurales et urbaines, ni du degré de l'influence réelle de l'Église sur les opinions ou les habitudes des diverses classes sociales ${ }^{15}$ ». Son propre inventaire le conduisait alors à recenser une cinquantaine de titres et il semblait bien qu'il s'agissait là de la totalité de la production en sociologie religieuse au Canada français. En 1956, Louis-Edmond Hamelin et Colette L. Hamelin dressaient une liste de près de 300 travaux dans le même domaine ${ }^{16}$. Louis-Edmond Hamelin allait lui-même contribuer de manière importante à la discipline en publiant, quelques années plus tard, une importante recherche sur l'évolution numérique du clergé québécois dans la deuxième parution de Recherches sociographiques ${ }^{17}$.

Ce n'est que progressivement - mais les évolutions seront rapides au cours des années 1950 - que les études de sociologie religieuse se développent, venant ainsi combler le vide constaté par Falardeau en 1951. On doit les premières enquêtes à Vianney Delalande et au père Motte, tous deux franciscains, qui les menèrent au cours de missions intérieures. En

15. J.-C. Falardeau, "Les recherches de sociologie religieuse au Canada», dans Milieux modernes et Vie religieuse, numéro spécial de la revue Lumen Vitae, VI (1951) : 139.

16. Pour une liste des travaux en sociologie religieuse au Canada, avant 1956, voir L.-E. Hamelin et C.-L. Hamelin, Quelques matériaux de sociologie religieuse canadienne (Montréal, Les éditions du Lévrier, 1956).

17. L.-E. Hamelin, "Évolution numérique séculaire du clergé catholique dans le Québec», Recherches sociographiques, 2 (1960): 220. 
1958, le chanoine Boulard, formé par Gabriel LeBras, dirige lui-même une enquête dans le diocèse de Québec, dans la région de Lotbinière. Il initie quelques curieux en la matière à ses outils et à ses méthodes de recherche. À cette occasion, il donne quelques leçons au Grand Séminaire de Québec et expose ses résultats à l'occasion d'une séance tenue à la salle des Promotions du Séminaire ${ }^{18}$.

C’est cette même année qu'est fondée l'Association québécoise de sociologie religieuse. Fernand Dumont en devient le président et Roland Doyon le secrétaire. Ce dernier, prêtre du diocèse de Québec, avait déjà étudié la sociologie, à partir de 1953, à l'instigation de $M^{\text {gr }}$ Ernest Lemieux, désireux de le voir enseigner la sociologie au Grand Séminaire. Il enseignera finalement en sciences sociales à l'Université Laval — avec un cours à la Faculté de théologie - et fondera, en 1958, le Centre de recherches en sociologie religieuse de l'Université Laval qui conduira par la suite un grand nombre d'analyses portant sur la situation religieuse dans le diocèse de Québec.

La création de ce Centre de recherche en sociologie religieuse n'est qu'un indicateur d'un phénomène plus vaste : l'entrée des sciences humaines et sociales dans le champ de la théologie et de la pastorale. On aura noté que cette fondation intervient la même année que la création, à la même faculté, de l'Institut de pastorale et deux ans avant la fondation de l'Institut de catéchèse. Il ne s'agit pas là de simples coïncidences et il faut voir la connexion étroite entre ces trois fondations. La lecture des buts poursuivis par le Centre de sociologie religieuse est explicite :

- Il veut faire avancer la sociologie religieuse de notre milieu et servir de base expérimentale à l’enseignement de la théologie et de la pastorale.

- Il est une sorte de laboratoire au service de la théologie et de la pastorale ${ }^{19}$.

La sociologie était donc considérée comme une science auxiliaire qui devait être mise au service des pasteurs de manière à mieux orienter leur action pastorale. Le cours offert en 1964 par Roland Doyon, «Sociologie religieuse au service de la pastorale », illustre bien la relation que l'on établit entre les deux termes. La sociologie, au même titre que la psychologie pour la catéchétique, était mise au service de l'action pastorale. La

18. On trouve à la bibliothèque de l'archevêché le programme de cette "grande semaine d'études pastorales» tenue à la salle des Promotions du 9 au 14 février 1959.

19. Annuaire de la Faculté de théologie, 1959. 
pastorale et la catéchèse, avec le présupposé qu’elles tiraient toutes les conséquences pratiques de la théologie, étaient alors conçues comme des arts faisant appel à des savoir-faire. Elles devaient donc s'appuyer sur les sciences humaines (psychologie) et sociales (sociologie) qui leur procuraient une expertise à ce niveau. À l'Université Laval, c'est dans les cadres de ces deux instituts (pastorale et catéchèse) que seront intégrés les professeurs qui auront une formation en sociologie et en psychologie $e^{20}$.

Ce premier frémissement à la Faculté de théologie correspond à la période des nombreuses enquêtes, menées sur une grande échelle, et qui prendront la forme de Grandes missions diocésaines. C'est ainsi que, de retour à Québec en 1962 après un séjour en Europe pour des études doctorales sous la direction de Fernand Boulard, R. Doyon conduira la Grande mission et sera le maître d'œuvre de la pastorale d'ensemble au diocèse de Québec et de la réforme des institutions du gouvernement du diocèse au cours de l'après-concile. Ce n'est qu'à ce moment que commencent vraiment, dans le diocèse de Québec, des enquêtes systématiques de sociologie religieuse, selon la méthode du contact global qui conduisait à une perception plus fine des nouveaux milieux humains qui se sont constitués au cours de l'après-guerre.

Dans l'intervalle cependant, les travaux avaient déjà été amorcés aux diocèses de Montréal avec Louis Bovy ${ }^{21}$, de Saint-Jérôme, sous la direction de Fernand Dumont et Yves Martin ${ }^{22}$, et de Saint-Jean (Longueuil) avec Jean-Guy Bissonnette. Des contacts fréquents ont lieu entre Maurice Matte de Saint-Jérôme et Jean-Guy Bissonnette de Saint-Jean et Roland Doyon de Québec ${ }^{23}$. Il ne s'agit donc pas d'initiatives isolées, mais d'un mouvement

20. Les abbés Jean-Marie Lachance (L. psychologie expérimentale (Montréal), professeur à l'Institut de pastorale, 1964-1966) et Armand Maranda (L. en orientation professionnelle, professeur à l'Institut de catéchèse et à l'Institut de pastorale de 1964-1966) donnaient aux séminaristes des enseignements en psychologie de la vocation depuis 1959. Par la suite (1965-1966), PaulEugène Couture et Réginald Richad étaient appelés à donner des enseignements en psychologie, respectivement à la Faculté de théologie et à l'Institut de catéchèse. Depuis la création de l'Institut de catéchèse, les enseignements en psychologie avaient été assurés par des professeurs étrangers, notamment Jean Le Du et le père Godin de Louvain.

21. Bien vite, des prêtres du diocèse de Montréal formés dans les sciences sociales prendront la relève. D’abord, Norbert Lacoste et Jean-Marie Lafontaine et, par la suite, Jean-Pierre Duchesne. De son côté, Jean-Claude Turcotte rentre de Lille diplômé dans le domaine de la pastorale sociale.

22. Jacques Grand'Maison en est alors à ses premières armes. Bientôt, il s'imposera comme un membre éminent de ce groupe de clercs formés aux sciences sociales.

23. Doyon sera vite suivi par une autre génération de prêtres formés dans les sciences sociales, à Rome ou à Laval : Pierre Morissette, Jean Picher, Michel Stein et Jacques Racine. 
où chacun profite des analyses, des hypothèses et des expériences des autres. Peu à peu, on avance vers la définition d'une pastorale d'ensemble qui modifiera les formes de gouvernement des diocèses du Québec ${ }^{24}$. Ce qu'il importe de noter ici, ce n'est pas simplement le fait que le renouveau de la pastorale et des institutions ecclésiales est surtout orienté par des enquêtes sociologiques, mais de voir que le travail des sociologues dans le champ ecclésial correspond au travail qu'ils accomplissent dans la sphère sociale et politique.

En effet, le travail effectué dans le diocèse de Saint-Jérôme dans le cadre de la Grande Mission, en 1958, sous la conduite des sociologues de l'Université Laval (Yves Martin et Fernand Dumont) — ceux-là mêmes qui ont amplement contribué à la formation des cadres qui allaient bientôt être aux commandes de l'État québécois - est homogène à celui qui s'amorce, à la même époque, dans le cadre de la planification de l'État québécois qui s'adosse, lui aussi, à de grandes enquêtes. La méthode qu'ils développent alors et qu'ils présentent dans leur ouvrage, L'analyse des structures sociales régionales, est à l'origine de trois grands chantiers ${ }^{25}$. Le premier, dans le domaine ecclésial, correspond aux Grandes Missions : Saint-Jérôme en 1958, Montréal et Saint-Jean (Longueuil) en 1960, Sainte-Anne-de-la-Pocatière et Chicoutimi en 1962, puis finalement Québec, en 1963. Le second, dans le domaine public, avec les grandes études du Bureau d’aménagement. Enfin, les étudiants du département de sociologie de l'Université Laval participeront à diverses recherches sur le terrain à partir du même modèle d'enquête et d'analyse en l'appliquant particulièrement à la régionalisation de l'enseignement secondaire qui s'est traduite par l'établissement des polyvalentes. On est donc en présence, au cours de la période conciliaire et des années qui suivent, d'une série d'interactions croisées entre le processus de régionalisation mis en avant par le gouvernement du Québec et la réorganisation des diocèses sur la base de la création de régions pastorales et la mise sur pied de nouveaux organismes centraux de gouvernement. Il importe de prendre les choses une à une si l'on veut mieux saisir les influences réciproques de ces divers phénomènes qui se présentent au même moment.

$\mathrm{Si}$, par leur appellation, elles semblent tirer leur inspiration de la tradition antérieure des missions paroissiales, les Grandes Missions du tournant des années 1960 procèdent pourtant d'une autre logique. Elles se

24. Voir sur le sujet G. Routhier, Les pouvoirs dans l'Église. Étude du gouvernement d'une Église locale : l'Église de Québec (Montréal, Médiaspaul, 2003), spécialement la quatrième partie.

25. F. Dumont et Y. Martin, L'analyse des structures sociales régionales (Sainte-Foy, Les Presses de l'Université Laval, 1963). 
distinguent non seulement par leur durée (celle du diocèse de Québec durera sept ans), mais elles se démarquent surtout par le fait que leur base géographique n'est plus la paroisse, mais une région sociologique homogène, et par l'importance et la nouveauté des moyens mis en œuvre. Une Grande Mission a pour but avoué d'aboutir à un plan pastoral unifié ou d'implanter une pastorale d'ensemble.

Les méthodes de travail adoptées sont similaires à celles mises en œuvre par l'État québécois au début des années 1960 : vastes enquêtes qui conduisent à la rédaction de volumineux rapports à partir des mêmes outils d'analyse, travail intense d'animation dans les régions, utilisation des sciences humaines et des nouvelles techniques de communication. Bref, la pastorale d'ensemble se sent bien à l'aise avec la nouvelle culture administrative de l'État. Ne la définit-on pas comme la «coordination de tous les efforts de l'Église diocésaine : coordination au niveau de la vie et coordination au niveau de la direction. C'est-à-dire, d'une part, planification de toutes les initiatives entreprises par chacun des grands secteurs de la pastorale [...]; et d'autre part, intégration de toutes ces initiatives dans un plan ordonné par un comité de direction ${ }^{26} . .$. . À terme, on doit arriver à un plan.

Les enquêtes dirigées dans les diocèses par des sociologues décrivent dans le détail le milieu socioreligieux et dressent aussi un tableau général de la situation de l'Église dans ce milieu spécifique (institutions, personnel, questions et inquiétudes des fidèles en regard de la foi, etc.). Elles comportent aussi une partie prospective en formulant quelques suggestions et quelques priorités pastorales. Pour la constitution de ces dossiers, on recourt à différentes formes d'animation populaire : on regroupe des prêtres et des laïcs de toute une région, afin de colliger leurs réflexions. Par la suite, les résultats de ces enquêtes sont communiqués dans le cadre de soirées sociologiques auxquelles participaient les responsables de la vie socioparoissiale d'une région. Enquête, participation, animation sociale sont les maîtres-mots de cette expérience.

Ce qu'il importe de remarquer, c'est que ce mouvement est tout à fait contemporain des premières expériences de planification économique et de régionalisation mises de l'avant par le gouvernement Lesage, en 1961 à la suite de l'adoption de la loi sur le Conseil d'orientation économique du Québec qui avait notamment pour mission de préparer un plan de développement économique du Québec ${ }^{27}$. Si cet organisme et ceux qui

26. Claude Bélanger, «Le Conseil diocésain de pastorale», Église de Québec, 14 avril 1966.

27. Ce conseil sera remplacé, en 1968, par deux organismes, l'Office de planification et de développement du Québec et le Conseil de planification et de développement du Québec. 
l'ont remplacé ont travaillé des dossiers sectoriels (par exemple sur l'électricité et la sidérurgie), ils se sont surtout consacrés à des dossiers régionaux (Bas-Saint-Laurent, Saguenay-Lac-Saint-Jean, Nord-ouest du Québec, etc. ${ }^{28}$. On vise alors l'aménagement régional et l'aménagement global du territoire. L'expérience la plus poussée d’aménagement régional est celle qui s'est déroulée dans l'Est du Québec sous les auspices du Bureau d'aménagement de l'Est du Québec, organisme qui avait pour but, à partir de 1963, de préparer un plan de développement régional.

Les Grandes Missions correspondent donc exactement à la période faste des conseils économiques régionaux et des conseils régionaux de développement. La description de cette vaste expérience d’animation régionale qu'ils mettent en œuvre ressemble à s'y méprendre à celles que l'on donne des Grandes Missions régionales à la même époque ${ }^{29}$.

Les recherches et mémoires qui conduisent à l'élaboration d'une pastorale d'ensemble dans le diocèse de Québec entre 1963 et 1966 comportent les mêmes caractères que ceux que l'on retrouve dans les documents produits, à la même époque, par les conseils de développement régional. Les termes "plan pastoral ${ }^{30}$ », "pastorale d'ensemble» et «coordination ${ }^{31}$ »

28. Sur l'expérience de la planification au Québec, voir J. I. Gow, Histoire de l'administration publique québécoise, 1867-1970 (Montréal, Presses de l’Université de Montréal, 1986), 302-307; J. Benjamin, Planification et politique au Québec (Montréal, Presses de l'Université de Montréal, 1974); A. Baccigalupo, «Administrations publiques territoriales et planification régionale dans la province de Québec», Revue administrative, XXV (1972) : 61-65 ; J. Leblanc, «Le conseil d’orientation économique du Québec», Relations industrielles, XVIII,1 (1963) : 110-120; M. Lord et G. Daoust, «Le gouvernement du Québec et la planification ", La Presse, 13, 14 et 15 mars 1969; R. Parenteau, "L’expérience de la planification au Québec», Actualité économique, XXV,4 (1970) : 679-696.

29. Pour une description de l'animation régionale qui doit conduire à l'élaboration des plans de développement régionaux, voir J. I. Gow, op. cit., 308.

30. Voir par exemple ces extraits d'un texte de Roland Doyon décrivant les objectifs de la Grande Mission : elle veut «établir une pastorale d'ensemble qui, au niveau régional, grouperait toutes les forces de l'Église de Charlevoix autour de l'Évêque, les coordonnerait et les orienterait selon un plan d'évangélisation». Plus loin : "Avant d'œuvrer sur le terrain et d'établir un plan d'évangélisation, il fallait prendre une vision synthétique et explicative [...], de l'univers socioreligieux en cause». R. Doyon, «La Grande Mission de Charlevoix», La Semaine religieuse de Québec, 41 (11 juin 1964) : 654-658.

31. Le terme "coordination» ou ses dérivés reviennent à neuf reprises en deux pages, dans un projet de circulaire au clergé leur annonçant la création du nouveau conseil diocésain de pastorale. Il se marie bien avec ces autres termes incantatoires que sont "planifier» et "concerter» auxquels il se mêle dans le même paragraphe. Dans le premier projet de circulaire, il est employé quatre fois en deux pages. Voir "Projet de circulaire», $1: 4-5 ; 2: 2-3$, AAQ, 1J. Le terme "coordination» est celui qui revient le plus fréquemment dans les documents relatifs au Conseil diocésain de pastorale entre 1963 et 1966. On le trouve dix fois en neuf pages dans le «Mémoire Doyon» et six fois en trois pages dans un article de Claude Bélanger. 
s'imposent et correspondent aux «plans de développement régional» ou aux "plans d'ensemble». Comme dans l'appareil d'État, des institutions centrales visant la coordination de «toutes les forces vives de l'Église» sont mises en place ${ }^{32}$.

On l'aura deviné, cette planification d'ensemble et cette coordination de tous les efforts ne s'acquièrent qu'au prix d'une certaine centralisation. Elle connait deux formes principales : de larges secteurs de la pastorale qui n'étaient pas coiffés jusque-là d'un bureau diocésain se voient maintenant rattachés à un office diocésain, tous les secteurs de la pastorale étant désormais pris en charge par l'administration diocésaine. On remarque aussi l'intervention plus accusée des organismes centraux dans la vie pastorale des paroisses, des régions, des organismes, associations et mouvements. On assiste à une véritable intégration verticale de l'activité pastorale.

Ce n'est pas seulement sur le plan de la reconfiguration des institutions centrales de gouvernance que les Églises du Québec imitent l’administration publique. Le même phénomène de régionalisation est observable dans les deux institutions. Le mouvement de régionalisation du diocèse de Québec, par exemple, s'amorce au moment où l'administration publique est ellemême saisie d'une fièvre de régionalisation. C'est la période de la création des Commissions scolaires régionales avec le lancement, en 1964, de l'opération 55 et de la mise sur pied des Conseils régionaux de santé et de services sociaux, bientôt suivis des municipalités régionales. Le 29 mars 1966, un arrêté-en-conseil divise le Québec en dix régions administratives, établit sept métropoles régionales et institue dix-huit centres sous-régionaux ${ }^{33}$.

Dans les diocèses, on retrouve le même mouvement de régionalisation, de planification et de centralisation. L'organisme central de planification et de coordination de la pastorale, le Conseil diocésain de pastorale (CDP), a la responsabilité de mettre sur pied des structures régionales et des offices curiaux nécessaires à l'établissement d'une pastorale d'ensemble. "Par rapport aux Commissions de secteurs... le rôle du CDP est de les fonder, de les faire travailler et de les coordonner. Quant aux régions, le CDP aura

32. En 1962, constitution d’une Commission générale de pastorale dont dépendent le Secrétariat de la Grande Mission et le Comité diocésain d'action catholique; en 1963, création du Conseil diocésain de pastorale; en 1968, mise sur pied d'un Secrétariat de la pastorale d'ensemble; en 1969, création du Service de la pastorale et, en 1970, du Comité des organismes et du Comité des régions.

33. Il s'agit de l'arrêté 524. Cette réforme de la carte administrative avait commencé en 1961 au ministère de l'Industrie et du Commerce avec la création de 14 territoires de développement industriel. Voir A. Baccigalupo, loc. cit., 61-65. On complétera en consultant J. I. Gow, op. cit., 247-253. 
à les constituer en zonant le diocèse, à organiser la vie à l'intérieur de chacune et à les coordonner entre elles ${ }^{34}$.»

Ces phénomènes, dans l’Église de Québec et dans l’État québécois, sont non seulement exactement concomitants, mais les deux réformes procèdent des mêmes logiques, sont pilotées par des experts formés aux mêmes écoles, induisent les mêmes effets et appartiennent à la même culture administrative. En fait, cela n'est pas surprenant lorsqu'on considère le va-et-vient du personnel entre l'administration gouvernementale et l'administration ecclésiale, spécialement par le biais du ministère de l’éducation qui représente le type même de la nouvelle administration publique centralisée et planificatrice.

À la suite de Vatican II, le diocèse de Québec adoptait le modèle de la pastorale d'ensemble, redéfinissait les organismes centraux de son gouvernement, établissait un plan de régionalisation et procédait à une réforme de ses procédures administratives, et cela, au moment où l'État québécois renouvelait ses organismes administratifs centraux, procédait à la planification et au développement régional et modernisait ses procédures administratives. Il n'y a pas seulement synchronie, mais développement symétrique. Nous retrouvons les mêmes logiques d'action, des institutions au profil semblable, des procédures et des méthodes de travail apparentées et un même jargon administratif. En somme, l'influence de la bureaucratie étatique dépasse dans son rayonnement sa propre sphère d'activités, comme le souligne en conclusion James I. Gow pour qui la progression de l'administration publique

oblige les institutions et les groupes qui traitent avec l'administration à se bureaucratiser à leur tour [...]. Les services publics décentralisés sont bouleversés par leurs contacts avec la bureaucratie provinciale. La bureaucratisation apparaît aussi au niveau des corps intermédiaires qui doivent se structurer en vue d'échanges avec l'administration. Cela se voit de manière très nette : ils envoient des représentants au sein des organismes consultatifs, et les contacts directs doivent être précédés d'études et de recherches donnant lieu normalement à des mémoires ou autres documents de ce genre ${ }^{35}$.

Il y a donc eu, au cours de cette période, une véritable rencontre, sinon une fusion, entre sociologie et théologie, rencontre qui marquera durablement la théologie québécoise. Cette embrassade des deux disciplines connaît

34. «Mémoire Doyon», 4. Le souligné est dans l'original. Voir également "Conseil diocésain de pastorale. Rapport global de ses activités depuis ses débuts", AAQ, 1J.

35. J. I. Gow, op. cit., 364. 
probablement son sommet en 1971, au moment de la publication du rapport de la Commission Dumont, commission d'études sur les laïcs et l'Église, présidée par le sociologue de Laval et dont deux commissaires seront des prêtres sociologues, Jacques Grand’Maison et Jean-Marie Lafontaine.

\section{UN RÉÉQUILIBRAGE DANS LE RESPECT MUTUEL}

Il ne faut pas croire que le recours à la sociologie dans le champ pastoral ne suscite que de l'enthousiasme. De part et d'autre, on ressent un certain inconfort. Inconfort, d'abord, de la sociologie pastorale qui subit de vives critiques des sociologues de la religion. On n'a qu'à se rappeler le commentaire d’Émile Poulat adressé au célèbre projet inauguré en 1931 par Gabriel Le Bras et que son collègue a désigné comme "un monument de style composite, clérico-universitaire ", d’où il résulte, dans le clergé, une image très appauvrie de la sociologie religieuse, qu'il réduit spontanément aux techniques qui lui sont devenues familières pour satisfaire à ses préoccupations propres $^{36}$. On reproche surtout à la sociologie pastorale d'être au service de l'institution religieuse et d'avoir pour seule finalité l'orientation de l'apostolat et l'affinement des stratégies pastorales. Inconfort aussi de la théologie, car les critiques viendront bientôt du côté de la théologie qui connaitra une évolution importante à partir des années $1960^{37}$ et qui sent bien le risque d'être dominée par les sciences humaines et assimilée aux sciences des religions qui connaissent une montée en puissance.

C'est dans ce contexte qu'un certain rééquilibrage verra le jour. D’une part, on assiste, non sans difficulté cependant, à la création de programmes autonomes en sciences des religions. Le mouvement s'amorce à l'Université Laval au début des années 1970, encouragé par les membres du Centre de sociologie religieuse (avec Jean-Paul Rouleau et Raymond Lemieux, en particulier) et s'achève, pour ainsi dire, à la fin des années 1990, au moment où l'on dispose de programmes en sciences des religions aux trois cycles. Cela se manifeste également par l'élargissement des appellations des facultés de théologie qui intègrent dans leur désignation ce volet distinct de formation. Les épistémologies sont de plus en plus distinguées et les amalgames ruineux sont évités. De son côté, la théologie connaît elle aussi une évolution sensible. Traditionnellement reconnue comme un discours déductif, la théologie

36. Émile Poulat, «Trois problèmes pour la sociologie du catholicisme», Social Compass, XVI,4 (1969) : 479-480.

37. L'abandon du thomisme, à la suite de Vatican II, met la théologie en déséquilibre et à la recherche de nouvelles références, au moment même où les sciences sociales triomphantes sont tentées de lui en donner de nouvelles. 
se présente de plus en plus comme un discours contextuel, souvent inductif, et qui porte une attention plus grande aux pratiques, si bien qu'elle intègre à sa démarche des parcours faisant appel aux sciences humaines et sociales. Conçue comme science herméneutique, elle a fini par accorder aux pratiques un statut qui leur était inconnu avant Vatican II.

Si aujourd'hui les frontières sont beaucoup mieux dessinées entre théologie et sciences sociales et si les relations sont de plus en plus respectueuses des limites propres à chacun de ces discours, les rapports parfois complexes entre ces deux mondes sont encore d'actualité et refont périodiquement surface. On l'a vu, en France, au moment de la publication de l'ouvrage Les Français sont-ils encore catholiques ${ }^{38}$ ou, plus récemment, lors du débat entourant la publication du volume de $\mathrm{M}^{\mathrm{gr}}$ Hyppolite Simon, Vers une France païenne. Quoi qu'il en soit, les acquis de plusieurs décennies de relations sont indéniables et ces deux disciplines s'instituent désormais dans la reconnaissance l'une de l'autre.

Par ailleurs - en dehors des universités cette fois - se développe, au cours de cette période, un autre lieu de rencontre et de transactions entre la théologie et les sciences sociales : il s'agit du terrain de l'engagement social. À partir de 1970, en particulier, les analyses sociales développées par les sciences sociales ont un réel impact sur les chrétiens engagés dans le domaine social et communautaire. Les centres de formation populaire (le centre Justice et foi des jésuites et le Centre Saint-Pierre des oblats, par exemple) illustrent bien l'impact qu'exerce la critique sociale développée par les sciences sociales sur les militants chrétiens. Outre ces deux centres montréalais, cette influence s'observe également dans ce qui reste de l'Action catholique (la Jeunesse ouvrière catholique et le Mouvement des travailleurs chrétiens), dans la Revue de vie ouvrière et dans le réseau de "Politisés chrétiens» qui apparaît au cours des années 1970, ou dans les communautés ecclésiales de base qui apparaissent au cours de la même période. Cette influence est aussi manifeste dans d'autres réseaux, notamment le Centre d'animation pastorale en milieu ouvrier de Québec, les offices de pastorale sociale des diocèses du Québec, l'organisme de solidarité internationale Développement et Paix mis sur pied par l'épiscopat en 1967, etc. Plus récemment, en 1991, cette influence est visible dans la relance des Journées sociales du Québec.

Cette influence de la critique sociale développée dans les sciences sociales n'est pas manifeste seulement dans les réseaux de militants chrétiens. On

38. Guy Michelat, Julien Potel et Jacques Sutter et al., Les Français sont-ils encore catholiques? Analyse d'un sondage d'opinion (Paris, Cerf, 1991). 
la retrouve en particulier dans les documents des évêques du Québec, en particulier leur message du $1^{\mathrm{er}}$ mai. Il y a donc là un autre niveau d'interactions entre théologie et sciences sociales.

\section{CONCLUSION}

Dans son panorama des sciences des religions, Jean Seguy identifiait lui aussi trois phases dans l'évolution des rapports entre théologie et sciences des religions. Il présentait l'histoire de ces rapports à partir de la métaphore d'une "querelle de génération à l'intérieur d'une famille ${ }^{39}$ ». Dans un premier temps, celui des mères abusives, la mère théologie aurait "tenté d'empêcher ses filles, les sciences laïques, de prendre leur envol». Cette première phase aurait été suivie d'une deuxième, celle des vierges folles, qui représente un temps d'émancipation où les sciences humaines et sociales abandonnent, en la contestant, leur matrice théologique et campent hors de la cité théologienne. À cette deuxième phase suit une troisième, celle des mères repenties et des filles prodigues, caractérisée par l'abandon des polémiques, et qui entraîne, d'une part, la théologie à emprunter des méthodes et des approches élaborées par les sciences humaines et sociales et, d'autre part, les sciences humaines et sociales à ne pas prétendre expliquer sans reste le fait religieux.

Cette façon imagée de décrire les relations entre ces deux univers des sciences ne décrit pas exactement les évolutions que l'on peut repérer ici, le Québec n'ayant pas connu le même cheminement que la France quant à la place de la religion dans le cadre de l'université publique et pluraliste. Toutefois, cette description demeure suggestive et montre bien des relations complexes, parfois polémiques, mais qui tendent aujourd'hui à un nouvel équilibre correspondant à ce que nous avons appelé, à l'Université Laval, une fécondation croisée de ces deux approches disciplinaires du fait religieux.

39. Jean Séguy, "Panorama des sciences des religions», dans Henri Desroche et Jean Séguy, Introduction aux sciences humaines des religions (Paris, Cujas, 1970), 37-52. 